\title{
A CLASS OF SELF-SIMILAR RANDOM MEASURE
}

\author{
DAVID VERE-JONES, ${ }^{*}$ Victoria University and Statistical Research Associates, Ltd
}

\begin{abstract}
We describe a class of self-similar random measure that generalizes the class of stable, completely random measure, or, in one dimension, the class of processes with stable independent increments. As with the stable processes, the realizations are purely atomic, but the masses of the atoms are not necessarily independent, but rather characterized by self-similar dependence relations. Indeed, the class can be described most effectively in terms of the point process on the product space for the locations and sizes ('marks') of the atoms. Then self-similarity reduces to an invariance relation ('biscale invariance') on the distribution of this marked point process. The condition can be satisfied when the marked point process is compound Poisson, corresponding to the nonnegative stable processes, but is by no means restricted to this case. An example is given which modifies and extends Ogata's epidemic-type aftershock sequence model for earthquake occurrence.
\end{abstract}

Keywords: Self-similar process; random measure; point process; Lévy process

2000 Mathematics Subject Classification: Primary 60G18; 60G55; 60G57

\section{Introduction}

In this note, we consider a class of purely atomic random measures which are stationary, self-similar, but more general than the stable random measures in that they admit dependence relations between the sizes and locations of the atoms.

The underlying idea of self-similarity is that a change in scale is balanced by a compensating change in mass. We adopt the following definition (see Lamperti (1962), Vervaat (1985), and Daley and Vere-Jones (1988, p. 326, Example 10.1(f)), writing $\mathbb{R}^{d}$ and $\mathbb{B}^{d}$ for $d$-dimensional Euclidean space and its Borel $\sigma$-algebra, respectively, and $\mathbb{R}^{+}$for the half-line $(0, \infty)$.

Let $H$ be a finite positive constant. A random measure $\xi(\cdot)$ on $\mathbb{R}^{d}$ is said to be self-similar, with similarity index $H$, if its distributions are invariant under the group of transformations (or renormalization group)

$$
R_{a}^{H} \xi(A)=a^{-H} \xi(a A), \quad A \in \mathbb{B}^{d}, a \in \mathbb{R}^{+} .
$$

In most cases, we require the random measure to be stationary (invariant under shifts in $\mathbb{R}^{d}$ ) as well. In the one-dimensional case, the two conditions together are equivalent to requiring the cumulative process $X(t)=\int_{0}^{t} \xi(\mathrm{d} s)$ to have stationary, self-similar increments.

As a random measure, a point process cannot be self-similar in this sense, if only because its values are integers, meaning that (1) cannot hold for all $a$. The best-known examples of stationary, self-similar random measures are the stable random measures, where $\xi$ is completely random (that is, the $\xi\left(A_{i}\right)$ are mutually independent whenever the $A_{i}$ are disjoint) and $\xi(A)$ has

Received 23 August 2004; revision received 31 August 2005.

* Postal address: Department of Mathematics, Victoria University of Wellington, Wellington, New Zealand. Email address: dvj@mcs.vuw.ac.nz 
a nonnegative stable (Lévy) distribution with Laplace transform of the form $\ell(s)=\exp \left(-c s^{\alpha}\right)$, for some $c>0$ and $\alpha=1 / H, 1<H<\infty$. The last restriction places the processes in a different group from the fractional Brownian motions, which are often used for models with multiplicative rather than additive independence and have $0<H<1$.

If we specialize to the case in which the measure has no fixed atoms and no drift, such completely random measures have a Lévy-Khinchin representation (see Kingman (1967) and Daley and Vere-Jones (1988, p. 177, Theorem 6.3.VIII)),

$$
\xi(A)=\int_{0}^{\infty} \kappa N(A \times \mathrm{d} \kappa) .
$$

Here $\kappa$ represents the size of the atom, while $N(\cdot)$ is a Poisson process on $\mathbb{R}^{d} \times \mathbb{R}^{+}$, the parameter measure $\mu$ of which satisfies, for bounded Borel subsets $A$ of $\mathbb{R}^{d}$ and all $\varepsilon, 0<\varepsilon<\infty$,

$$
\begin{array}{r}
\int_{\kappa>\varepsilon} \mu(A \times \mathrm{d} \kappa)<\infty, \\
\int_{0<\kappa<\varepsilon} \kappa \mu(A \times \mathrm{d} \kappa)<\infty,
\end{array}
$$

and, for all $x \in \mathbb{R}^{d}$,

$$
\mu(\{x\} \times(0, \infty))=0 .
$$

It should be noted that here and throughout the later discussion we need a slightly extended definition of a point process $N$ on $\mathbb{R}^{d} \times \mathbb{R}^{+}$that allows $N(A \times(0, \varepsilon))$ to be infinite even when $A$ and $\varepsilon$ are bounded. The essential requirement is that $N(A \times K)<\infty$ whenever $A$ and $K$ are bounded, and that $K$ also be bounded away from zero. This can be made compatible with the usual definition of local boundedness (bounded finiteness in Daley and Vere-Jones (1988)) by modifying the metric on $\mathbb{R}^{+}$so that the point 0 is at infinite distance from any positive $\kappa$, for example through the transformation $\kappa^{\prime}=\log \kappa$.

When the process is stationary on $\mathbb{R}^{d}, \mu$ reduces to the product $\mu=\ell \times \Psi$ of the Lebesgue measure $\ell$ on $\mathbb{R}^{d}$ and a $\sigma$-finite measure $\Psi$ on $\mathbb{R}^{+}$. If, in addition, the process is self-similar with similarity index $H$, then $\Psi$ takes the power law form

$$
\Psi(\mathrm{d} \kappa)=v \kappa^{-(1+1 / H)} \mathrm{d} \kappa,
$$

where $v$ is a finite positive constant, while, to satisfy (3) and (4), we require that $1<H<\infty$.

The main purpose of the present note is to point out that a considerably wider class of self-similar, stationary random measure can be obtained by retaining the representation (2) but dropping the requirement that $N(\cdot)$ be a Poisson process.

\section{Biscale invariance}

In establishing the power law form (5) when the completely random measure is self-similar, a key step is to show that invariance of the distributions of the original random measure under the transformations $R_{a}^{H}$ is equivalent to invariance of the Poisson point processes $N$ appearing in (2) under the group of biscale transformations $D_{a}^{H}: \mathbb{R}^{d} \times \mathbb{R}^{+} \rightarrow \mathbb{R}^{d} \times \mathbb{R}^{+}$, where

$$
D_{a}^{H}(x, \kappa)=\left(a x, a^{H} \kappa\right) .
$$

In general, if the distribution of a point process $N$ is invariant under the group $D_{a}^{H}$, we shall say that $N$ is biscale invariant with index $H$. Then the following lemma holds, irrespective of whether the process $N$ is Poisson. 
Lemma 1. Suppose that the random measure $\xi$ can be represented, as in (2), in terms of a point process $N$ on the product space $\mathbb{R}^{d} \times \mathbb{R}^{+}$. If, for given a and $H$, the distribution of $N$ is invariant under the transformation $D_{a}^{H}$, then the distribution of $\xi$ is invariant under the transformation $R_{a}^{H}$.

Proof. Consider the one-dimensional distribution of $\xi(A)$ for some bounded set $A \in \mathbb{B}^{d}$. Using ' $\stackrel{\text { D }}{=}$ to denote equality in distribution, we have

$$
\begin{aligned}
R_{a}^{H} \xi(A) & =\int_{0}^{\infty} \kappa a^{-H} N(a A \times \mathrm{d} \kappa) \\
& =\int_{0}^{\infty} \kappa N\left(a A \times \alpha^{H} \mathrm{~d} \kappa\right) \\
& \stackrel{\mathrm{D}}{=} \int_{0}^{\infty} \kappa N(A \times \mathrm{d} \kappa) \\
& =\xi(A) .
\end{aligned}
$$

Thus, the one-dimensional finite distributions of $\xi$ are invariant under $R_{a}^{H}$. For all $k>1$, similar arguments can be applied to the $k$-dimensional distributions of bounded Borel sets $\left(A_{1}, \ldots, A_{k}\right)$, at least when these are disjoint. However, since such finite-dimensional distributions are sufficient to determine the full distributions of $\xi$ and $R_{a}^{H} \xi$ completely, it follows that the two random measures must be equal in distribution.

Building on this result, we identify conditions on $N$ that will result in the associated random measure $\xi$ satisfying stationarity and self-similarity requirements. We assume throughout that $N$ defines a valid point process, in the extended sense already referred to, on the product space $\mathbb{R}^{d} \times \mathbb{R}^{+}$. We shall say that $N$ has finite expectation measure $M$ if $M(A \times K)=\mathrm{E}[N(A \times K)]$ exists and is finite for all bounded Borel sets $A$ of $\mathbb{R}^{d}$ and Borel sets $K$ of $\mathbb{R}^{+}$that are bounded away from the origin.

Proposition 1. (i) In order that (2) should define a valid (boundedly finite) random measure $\xi$ on $\mathbb{R}^{d}$, it is necessary and sufficient that, for all bounded $A \in \mathbb{B}^{d}$, the integral (2) should exist and be almost surely finite. If $N$ has finite expectation measure $M(\cdot \times \cdot)$, then it is sufficient for the existence of $\xi$ that $M$ should satisfy (3) and (4).

(ii) If $N$ is invariant under shifts in its first argument, then $\xi$ is stationary.

(iii) If $N$ is biscale invariant with index $H$, then $\xi$ is self-similar with similarity index $H$.

(iv) If the expectation measure $M$ exists and (ii) is satisfied, then there exists a $\sigma$-finite measure $\Psi$ on $\mathbb{R}^{+}$such that $M=\ell \times \Psi$, where $\ell$ is Lebesgue measure on $\mathbb{R}^{d}$. If (iii) is also satisfied, with index $H$, then $\Psi$ reduces to the form (5).

The proofs of these four statements are applications of standard results on the existence and convergence of integrals with respect to random measures: see, for example, Daley and Vere-Jones (1988, Chapter 6, pp. 155ff.).

These results provide a means of constructing self-similar random measures via the associated marked point processes. For one-dimensional (time) processes, such a point process is often most conveniently defined through its conditional intensity

$$
\lambda(t, \kappa) \mathrm{d} t \mathrm{~d} \kappa=\mathrm{E}\left[N(\mathrm{~d} t \times \mathrm{d} \kappa) \mid \mathscr{H}_{t}\right],
$$


where $\mathscr{H}_{t}$ is the history up to (time) $t$. It is therefore relevant to ask what conditions on $\lambda$ might lead to point processes satisfying the above conditions. In situations in which the underlying process is stationary and the history incorporates information from the indefinite past, the relevant conditional intensity was called a complete intensity by Daley and Vere-Jones (2003, Section 13.5, p. 558), and denoted by $\lambda^{\dagger}(t, \kappa)$; we adopt this terminology and notation. In what follows, we shall identify conditional expectations with respect to the history $\mathscr{H}_{t}$ with functions of the realization of the past of the process, that is, with functions on the space of counting measures on $(-\infty, t) \times(0, \infty)$ (which is justified by the fact that the counting measures themselves can be represented as points in a complete separable metric space). Also, we shall denote by $\left[T_{\tau} \mathscr{H}_{t}\right]$ and $\left[D_{a}^{H} \mathscr{H}_{t}\right]$ the histories associated with the process after transformation by the shift $T_{\tau}$ and the biscale transformation $D_{a}^{H}$, respectively, and adopt a similar interpretation for conditioning on $\left[T_{\tau} \mathscr{H}_{t}\right]$ and $\left[D_{a}^{H} \mathscr{H}_{t}\right]$. We then have the following result.

Proposition 2. (a) For $N$ to be stationary it is necessary that, for all real $t$ and $\tau$,

$$
\lambda^{\dagger}(t+\tau, \kappa) \mathrm{d} t \mathrm{~d} \kappa=\mathrm{E}\left[T_{\tau} N(\mathrm{~d} t \times \mathrm{d} \kappa) \mid T_{\tau} \mathscr{H}_{t}\right]=\lambda^{\dagger}(t, \kappa) \mathrm{d} t \mathrm{~d} \kappa .
$$

(b) For $N$ to be, in addition, biscale invariant with parameter $H$, it is necessary that, for all real $t$ and $a>0$,

$$
\lambda^{\dagger}\left(a t, a^{H} \kappa\right) a^{1+H} \mathrm{~d} t \mathrm{~d} \kappa=\mathrm{E}\left[N\left(\mathrm{~d}(a t) \times \mathrm{d}\left(a^{H} \kappa\right)\right) \mid D_{a}^{H} \mathscr{H}_{t}\right]=\lambda^{\dagger}(t, \kappa) \mathrm{d} t \mathrm{~d} \kappa .
$$

The first condition requires the conditional intensity to depend on the times of past events through the differences $t-t_{i}$ only. The two conditions together mean that the conditional intensity should be expressible in the form

$$
\lambda^{\dagger}(t, \kappa)=\kappa^{-(1+1 / H)} h\left(\left\{\frac{\kappa}{\kappa_{i}}, \frac{\left(t-t_{i}\right)^{H}}{\kappa_{i}}\right\}\right)
$$

for some function $h(\cdot)$ of a sequence of pairs, where $\left(t_{i}, \kappa_{i}\right)$ run through the sequence of points of the process with $t_{i}<t$, and the initial term in $\kappa$ arises from transformation of the infinitesimal elements $\mathrm{d} t \mathrm{~d} \kappa$.

The representation implies that, as in the Poisson case, the marginal distribution of the marks always has the form (5) and is invariant under time shifts. This distribution is not totally finite, but over any lower threshold $\kappa_{0}$ it can be normalized to form a Pareto distribution.

It should be noted that the conditions of Proposition 2 are not in general sufficient; for instance, it is easy to construct examples of processes for which part (a) holds yet the resulting process is explosive, meaning that no stationary version exists.

\section{A self-similar epidemic-type aftershock sequence model}

To exhibit an example different from the stable random measures, we construct a biscaleinvariant version of Ogata's epidemic-type aftershock sequence (ETAS) model, from which a self-similar random measure can be obtained by summing the marks (here interpreted as energies).

Recall that the standard ETAS model (see, e.g. Ogata (1988) or Daley and Vere-Jones (2003, p. 204, Example 6.4(d))) is a marked Hawkes process with complete conditional intensity, expressed in magnitudes $M$ rather than energies $\kappa=\mathrm{e}^{\theta M}$, and defined, for $M$ above some fixed threshold $M_{0}$, by

$$
\lambda^{\dagger}(t, M)=\beta \mathrm{e}^{-\beta\left(M-M_{0}\right)}\left\{\mu_{c}+A \sum_{\left\{i: t_{i}<t\right\}} \mathrm{e}^{\alpha\left(M_{i}-M_{0}\right)} f\left(t-t_{i}, c\right)\right\},
$$


where pairs $\left(t_{i}, M_{i}\right)$ refer to the times and marks of past events, $f(u, c)=p c^{p} /(c+u)^{1+p}$, and $A, p, c, \alpha$, and $\beta$ are positive constants. The condition for stability (i.e. the existence of a stationary version) is

$$
\rho \equiv A \int_{M_{0}}^{\infty} \mathrm{e}^{\alpha\left(M-M_{0}\right)} \beta \mathrm{e}^{-\beta\left(M-M_{0}\right)} \mathrm{d} M=\frac{A \beta}{\beta-\alpha}<1 .
$$

The variant of this model that we propose is defined by

$$
\lambda^{\dagger}(t, M)=\beta \mathrm{e}^{-\beta M}\left\{v+A \sum_{\left\{i: t_{i}<t\right\}} \mathrm{e}^{\beta M_{i}} f\left(t-t_{i}, c_{M_{i}}\right) S\left(M-M_{i}\right)\right\},
$$

where $c_{M}=\sigma \mathrm{e}^{\beta M}, S\left(M-M_{i}\right)=\mathrm{e}^{-\delta\left|M-M_{i}\right|}, \beta=\theta / H$, and $\nu, \eta, \sigma, p, \delta$, and $H$ are positive constants.

After converting to energies rather than magnitudes, we can express (7) in the form

$$
\lambda^{\dagger}(t, \kappa)=\kappa^{-(1+1 / H)}\left\{v+\eta \sum_{\left\{i: t_{i}<t\right\}}\left[1+\frac{t-t_{i}}{\sigma \kappa_{i}^{1 / H}}\right]^{-(1+p)} S\left(\log \left[\kappa / \kappa_{i}\right]\right)\right\} .
$$

The compatibility with (6) is now evident, but it is not clear whether there exists a welldefined process with this form of complete intensity.

To clarify this point, we make use of the cluster process representation of the Hawkes process (Hawkes and Oakes (1974), Daley and Vere-Jones (2003, p. 183, Example 6.4(c))). Our candidate model then has cluster centres forming a two-dimensional Poisson process with intensity $\psi(t, \kappa)=v \kappa^{-(1+1 / H)}$, while the cluster members from a parent at $\left(t_{c}, \kappa_{c}\right)$ are the total offspring, from all generations, of a branching process in which first-generation offspring form an independent Poisson process with intensity given by the kernel

$$
\theta\left(t, \kappa \mid t_{c}, \kappa_{c}\right)=\eta \kappa^{-(1+1 / H)}\left[1+\frac{t-t_{c}}{\sigma \kappa_{c}^{1 / H}}\right]^{-(1+p)} S\left(\kappa / \kappa_{c}\right), \quad t>t_{c}, \kappa>0 .
$$

The offspring from all later generations independently follow the same Poisson process relative to their own parent. Relative to the initial parent, the second generation follows a Poisson process with intensity

$$
\theta^{(2)}\left(t, \kappa \mid t_{c}, \kappa_{c}\right)=\int_{\left(t_{c}, \infty\right) \times \mathbb{R}^{+}} \theta\left(t, \kappa \mid t^{\prime}, \kappa^{\prime}\right) \theta\left(t^{\prime}, \kappa^{\prime} \mid t_{c}, \kappa_{c}\right) \mathrm{d} t^{\prime} \mathrm{d} \kappa^{\prime}
$$

and, in general, the $k$ th generation follows a Poisson process with intensity given by the $k$ th iterate of $\theta$, say $\theta^{(k)}$.

The expected number of offspring, from all generations, coming from a single ancestor (cluster centre) at $\left(t_{c}, \kappa_{c}\right)$ and falling into the set $B \times K$ is thus

$$
\sum_{k=1}^{\infty} \int_{B} \int_{K} \theta^{(k)}\left(\mathrm{d} t \times \mathrm{d} \kappa \mid t_{c}, \kappa_{c}\right)=\sum_{k=1}^{\infty} \theta^{(k)}\left(B \times K \mid t_{c}, \kappa_{c}\right),
$$

while the total expected number of points falling within $B \times K$, including cluster centres and all generations of offspring, is given by the integrated sum

$$
M(B \times K)=\sum_{k=0}^{\infty} \int_{\mathbb{R}^{d}} \int_{\mathbb{R}^{+}} \theta^{(k)}\left(B \times K \mid t_{c}, \kappa_{c}\right) \psi\left(t_{c}, \kappa_{c}\right) \mathrm{d} t_{c} \mathrm{~d} \kappa_{c},
$$


where $\theta^{(0)}$ is the identity kernel. However, it is not difficult to check that $\psi$ is an eigenvector of $\theta$ corresponding to the eigenvalue $\rho=\eta C / p \delta$. The sum in (9) therefore reduces to

$$
\nu\left(\sum_{k=0}^{\infty} \rho^{k}\right) \ell(B) \int_{K} \kappa^{-(1+1 / H)} \mathrm{d} \kappa,
$$

which is finite provided that $\rho<1, B$ is bounded, and $K$ is bounded away from the origin. It follows from standard theorems on the existence of Poisson cluster processes (see, for example, Daley and Vere-Jones (2003, p. 179, Proposition 6.3.III)) that the process exists and has finite first moment measure with density $m(t, \kappa)=[\nu /(1-\rho)] \kappa^{-(1+1 / H)}$.

If $\rho<1$ then the number of cluster members from a given ancestor is infinite in total, but most have very small marks and only a finite number fall into a bounded set $B \times K$ when $K$ is bounded away from the origin. On the other hand, the expected energy release from atoms in $B \times K$ remains finite provided that $K$ is bounded in the usual sense. Similar statements hold for the overall process.

\section{Extensions}

The model just described can be modified in many different ways without affecting either self-similarity or stationarity. Other types of shot noise process can be used in place of the ETAS model. For example, an exponential decay in time could be used just as well as the Pareto-type decay without affecting either stationarity or self-similarity. Similarly, for compatibility with the eigenfunction requirement, the stability factor $S\left(\kappa / \kappa^{\prime}\right)$ in (7) should satisfy $\int_{0}^{\infty}\left(1 / \kappa^{\prime}\right) S\left(\kappa / \kappa^{\prime}\right) \mathrm{d} \kappa^{\prime}=1$, but the minimum function is by no means the only example possible.

It seems more difficult to find alternatives to the self-exciting representation implied by (8), although it may be possible to find versions with no immigration component along the lines of Brémaud and Massoulié (2001).

We have limited the discussion in the present note to self-similarity in time, but similar branching process constructions can also be developed with self-similarity in space or spacetime (see Ogata (1998) for some options).

A related approach to self-similarity was initiated in papers by Zähle (1988, 1990a, 1990b, 1991), who suggested that the property be based not on absolute locations relative to a fixed origin in $\mathbb{R}^{d}$, but on locations relative to a given point of the realization, that is, on relative distances or time intervals between points. This amounts to examining the Palm distributions of self-similar random measures. Related ideas were explored in Chapter 7, Section 8, of Thorisson (2000). It would be of interest to explore these links more thoroughly. Another topic of interest would be the development of simulation routines for models of the kind considered here.

Finally, it seems plausible that any stationary, self-similar random measure must be purely atomic in character. This is certainly true for completely random measures; whether it remains true more generally appears to be an open question.

\section{Acknowledgements}

This note is one of several recent investigations which have their origins in the revision of Daley and Vere-Jones (1988). Daryl's stimulus and patience are gratefully acknowledged. I would also like to express my thanks to Yosi Ogata, Jiancang Zhuang, and Val Isham, among 
others, for their support and encouragement during the work on this note, and to anonymous referees whose comments helped considerably to clarify both its logic and its language.

\section{References}

Brémaud, P. And Massoulié, L. (2001). Hawkes branching point processes without ancestors. J. Appl. Prob. 38, 122-135.

Daley, D. J. And Vere-Jones, D. (1988). An Introduction to the Theory of Point Processes. Springer, New York.

Daley, D. J. And Vere-Jones, D. (2003). An Introduction to the Theory of Point Processes. Vol I. Elementary Theory and Methods, 2nd edn. Springer, New York.

Hawkes, A. G. And OAKes, D. (1974). A cluster representation of a self-exciting process. J. Appl. Prob. 11, $493-503$.

Kingman, J. F. C. (1967). Completely random measures. Pacific J. Math. 21, 59-78.

Lamperti, J. (1962). Semi-stable stochastic processes. Trans. Amer. Math. Soc. 104, 62-78.

OGAtA, Y. (1988). Statistical models for earthquake occurrence and residual analysis for point processes. J. Amer. Statist. Assoc. 83, 9-27.

Ogata, Y. (1998). Space-time point process models for earthquake occurrences. Ann. Inst. Statist. Math. 50, $379-402$.

Thorisson, H. (2000). Coupling, Stationarity, and Regeneration. Springer, New York.

VervaAt, W. (1985). Sample path properties of self-similar processes with stationary increments. Ann. Prob. 13, 1-27.

ZäHLE, U. (1988). Self-similar random measures. I. Notion, carrying Hausdorff dimension, and hyperbolic distribution. Prob. Theory Relat. Fields 80, 79-100.

ZäHLE, U. (1990a). Self-similar random measures. II. A generalization to self-affine measures. Math. Nachr. 146, 85-98.

ZäHLE, U. (1990b). Self-similar random measures. IV. The recursive construction model of Falconer, Graf, and Mauldin and Williams. Math. Nachr. 149, 285-302.

ZäHLE, U. (1991). Self-similar random measures. III. Self-similar random processes. Math. Nachr. 151, 121-148. 